Subscriber access provided by UNIV OF CAMBRIDGE

\title{
Article
}

\section{Evidence of stratification in binary colloidal films from microbeam X-ray scattering: Toward optimizing the evaporative assembly processes for coatings}

Amanda Carr, Weiping Liu, Kevin G. Yager, Alexander Francis Routh, and Surita Rani Bhatia ACS Appl. Nano Mater., Just Accepted Manuscript • DOI: 10.1021/acsanm.8b00968 • Publication Date (Web): 12 Jul 2018

Downloaded from http://pubs.acs.org on July 20, 2018

\section{Just Accepted}

"Just Accepted" manuscripts have been peer-reviewed and accepted for publication. They are posted online prior to technical editing, formatting for publication and author proofing. The American Chemical Society provides "Just Accepted" as a service to the research community to expedite the dissemination of scientific material as soon as possible after acceptance. "Just Accepted" manuscripts appear in full in PDF format accompanied by an HTML abstract. "Just Accepted" manuscripts have been fully peer reviewed, but should not be considered the official version of record. They are citable by the Digital Object Identifier (DOI®). "Just Accepted" is an optional service offered to authors. Therefore, the "Just Accepted" Web site may not include all articles that will be published in the journal. After a manuscript is technically edited and formatted, it will be removed from the "Just Accepted" Web site and published as an ASAP article. Note that technical editing may introduce minor changes to the manuscript text and/or graphics which could affect content, and all legal disclaimers and ethical guidelines that apply to the journal pertain. ACS cannot be held responsible for errors or consequences arising from the use of information contained in these "Just Accepted" manuscripts. 
Evidence of stratification in binary colloidal films from microbeam X-ray scattering: Toward optimizing the evaporative assembly processes for coatings

Amanda J. Carr ${ }^{1}$, Weiping Liu ${ }^{1}$, Kevin G. Yager ${ }^{2}$, Alexander F. Routh ${ }^{3}$, Surita R. Bhatia ${ }^{1 *}$

${ }^{1}$ Department of Chemistry, Stony Brook University, Stony Brook, NY USA

${ }^{2}$ Center for Functional Nanomaterials and National Synchrotron Light Source II, Brookhaven National Laboratory, Upton, NY USA

${ }^{3}$ Department of Chemical Engineering and Biotechnology, University of Cambridge, Cambridge United Kingdom

*Corresponding author, surita.bhatia@stonybrook.edu 


\begin{abstract}
Colloidal films have many important applications where a layered configuration is desirable, including flexible electronics, anti-reflective coatings, and anti-microbial paints. We report stratification during evaporative film formation in binary colloidal dispersion, probed using a novel microbeam small-angle X-ray scattering (SAXS) technique. To our knowledge, SAXS approaches have not been used to experimentally obtain quantitative data of concentration profiles in multicomponent colloidal films. We measured the local scattering of a film at different vertical locations using a microfocused X-ray beam and determined particle concentrations at different film depths using a linear combination analysis of the mixed film and pure film scattering data. Using small particle size ratios ranging from 2.55 to 1.25 , we experimentally observed and quantify three distinct stratification configurations: inverted smallon-top, large-on-top, and no stratification. Our results show some agreement with a previously proposed stratification state diagram, although there are some limitations. Experimental verification of these stratification phenomena is critical to fully understanding the physics of particle movement and structure development during film formation, which is crucial for optimizing evaporative assembly processes for coatings.
\end{abstract}

Keywords: SAXS, films, evaporative assembly, stratification, coatings, colloids 


\section{Introduction}

Considering the many applications of nanoparticle and colloidal films, absolute control over particle position during film formation is critical. Functionalized coatings are essentially films where a specific component with useful properties is desired at either the top or bottom of the film in a stratified configuration. Coatings can impart properties ranging from wettability, to glossiness, to deterred bacterial growth onto surfaces ${ }^{1-3}$. Controlled particle position during film formation is also applicable to inkjet printing, pesticide applications, flexible electronic device fabrication, production of paints and stains, and bulk heterojunction deposition in photovoltaics ${ }^{4-}$ ${ }^{12}$. Relevant to the model system of silica films presented in this work, the Velev group ${ }^{13}$ and Sunkara and coworkers ${ }^{14}$ have recently investigated anti-reflective coatings where the optical properties are dependent upon controlling the vertical concentration profile of silica nanoparticles within coatings. In all these examples, a single step deposition could greatly improve efficiency while reducing cost. However, particle movement during evaporative film formation in multicomponent systems is not well understood.

In films prepared by evaporation with only one particle size, the dimensionless Peclet number $(P e)$ balances particle diffusion and solvent evaporation, $P e=\frac{6 \pi \eta R H v_{e v}}{k T}$, where $\eta$ is the solvent viscosity, $R$ is the particle radius, $H$ is the initial film height, and $v_{e v}$ is the evaporation rate. Models have been developed based on $P e$ that predict non-uniform particle profiles during evaporation. ${ }^{15}$ The condition $P e>1$ predicts that large particles will jam at the air-film interface, as particle diffusion is slower than solvent evaporation. The condition $P e<1$ predicts a homogeneous distribution because particles can move through solution fast enough to randomly distribute before solvent evaporation causes jamming. These descriptions have been experimentally verified using specialized NMR profiling and cryogenic scanning electron 
microscopy ${ }^{16-17}$. Film stratification has also been quantified by scaling the particle concentration gradient between the top particle layer and edge of the film ${ }^{18}$ to $P e^{0.8}$.

In systems containing particles of different sizes, stratification is not as easily predicted.

Models of Trueman et al. that employ conventional colloidal hydrodynamics with arbitrary interactions have shown a large-on-top configuration, where the large particles become trapped by the moving air-solvent interface early within the film formation sequence ${ }^{19-20}$. However, even with some control of the large particle concentration at the top surface, experimentally it was often observed that an inverted small-on-top configuration formed. This has been investigated using theory, simulation, and microscopy experiments ${ }^{21-23}$. Theory of Zhou, Jiang, and Doi (hereby referred to ZJD) predicts small-on-top behavior in calculations that employ an expression for interparticle interactions similar to Trueman et al., but with an added cross-term to the free energy ${ }^{24}$. Fortini, Sear, and co-workers use different equations and include a surfaceinduced depletion term to predict a growing region rich in small particles at the top of the film ${ }^{21-}$ ${ }^{22}$. Recent modeling work by Sear ${ }^{25}$, building on results from Sear and Warren (hereby referred to as $\mathrm{SW})^{26}$, accounts for solvent flow and predicts both large-on-top and small-on-top stratification. This work also shows that stratification only occurs for large values of $P e$ of the small particles, and over a certain range of volume fraction of the small particles. However, these results are based on calculations performed for large size ratios, a different regime than the experimental system considered here. Finally, recent simulations by Cheng and co-workers ${ }^{27}$, performed over a wide parameter space of $P e$ and volume fraction, also show both small-on-top and large-on-top stratification and highlight the importance of including an explicit solvent in simulations. The three distinct types of possible stratification regimes are summarized in Figure 1. 


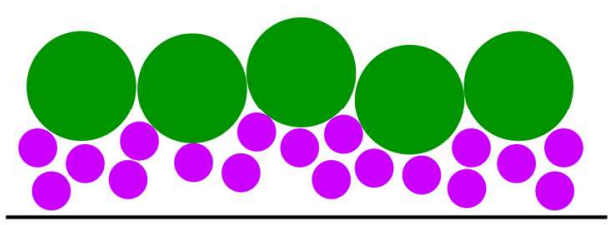

Large-on-top
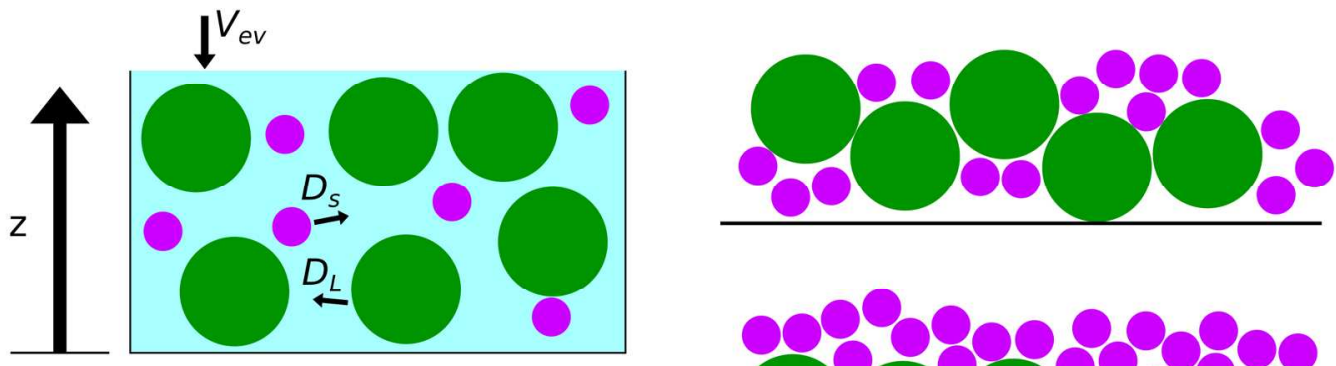

Not Stratified

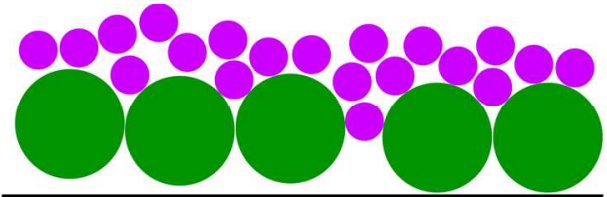

Small-on-top

Figure 1: Summary of observed particle configurations ${ }^{20-21,24}$.

Despite advances in theoretical descriptions and simulations of stratification, experimental measurement of particle concentration profiles throughout the film formation process has remained a challenge. Previous experimental studies have used NMR profiling, fluorescence tagging, and microscopy to determine particle locations ${ }^{16-21,28}$. However, all these methods are largely limited by small sample size areas, are restricted to specific particles types, and can be difficult to quantify. Here, we present an experimental investigation of local particle concentration at different film depths using a novel microbeam small angle X-ray scattering (SAXS) technique. We calculated the local particle concentrations of the films at different depths and present the results, along with comparison to recent theoretical descriptions of stratification.

\section{Experimental}

We prepared films using representative colloidal polystyrene and different sized silica particles. The hydrodynamic radii, as measured using dynamic light scattering (DLS), of the silica particles were: $8.41 \pm 1.1 \mathrm{~nm}(\mathrm{~S}-8), 9.91 \pm 0.46 \mathrm{~nm}(\mathrm{~S}-10)$, and $17.2 \pm 1.8 \mathrm{~nm}$, while the radius of the polystyrene was $21.4 \pm 1.2 \mathrm{~nm}$. Additional particle information is provided in Table 
1. Dispersions containing pure $3 \mathrm{v} / \mathrm{v} \%$ PS, pure $12 \mathrm{v} / \mathrm{v} \%$ silica, or a mixture of $3 \mathrm{v} / \mathrm{v} \%$ PS and $12 \mathrm{v} / \mathrm{v} \%$ silica were pipetted into plastic deep-well slides with a nominal diameter of $2.35 \mathrm{~cm}$ and height of $0.15 \mathrm{~cm}$, purchased from Diatec. Samples were then dried in an environmental chamber at $40.0{ }^{\circ} \mathrm{C}$ and $60 \%$ relative humidity overnight, as described in a previous work ${ }^{28}$.

To measure the evaporation rate, we pipetted a dispersion of $3 \mathrm{v} / \mathrm{v} \%$ silica into a deepwell slide and recorded the mass of the sample throughout the drying process. The mass of sample lost over time and the volume of the deep-well slide can then be used to calculate the velocity of the evaporating interface, also referred to as the rate of evaporation. For $3 \mathrm{v} / \mathrm{v} \%$ silica, the average rate of evaporation for three samples was $3.5 \times 10^{-8} \mathrm{~m} / \mathrm{s} \pm 0.3 \times 10^{-8}$. These data are supplied in the Supporting Information (Figure S1). Additional measurements for higher silica concentrations of $4-12 \mathrm{v} / \mathrm{v} \%$ silica showed only slight variation in the rate of evaporation, typically $<10 \%$ difference, within the uncertainty of the measurement. Thus, we assumed the rate of evaporation was the same for all pure silica, pure PS, and mixed silica and PS samples. We calculated the $P e$ values using the above rate of evaporation. These values are provided in Table 1.

The resulting films ranged from clear colorless to clear white, and some developed cracking fractures while drying. These cracks appeared late during the drying process. Dried film thicknesses were in the range 120-300 $\mu \mathrm{m}$.

Table 1: Summary of particles used

\begin{tabular}{ccccc}
\hline \hline Sample & $\begin{array}{c}\text { Hydrodynamic } \\
\text { Radius (nm)* }\end{array}$ & Polydispersity & $P e$ & $P e(\mathrm{PS}) / \mathrm{Pe}($ silica $)(\alpha)$ \\
\hline PS (PS-21) & $21.4 \pm 1.2$ & 0.024 & $3.2 \pm 0.2$ & --- \\
Silica (S-8) & $8.41 \pm 1.1$ & 0.165 & $1.3 \pm 0.2$ & 2.6 \\
Silica (S-10) & $9.91 \pm 0.46$ & 0.206 & $1.5 \pm 0.1$ & 2.2 \\
Silica (S-17) & $17.2 \pm 1.8$ & 0.128 & $2.6 \pm 0.3$ & 1.2 \\
\hline \hline
\end{tabular}

*Determined by dynamic light scattering. 
We collected SAXS data for each film at the CMS (11-BM) beamline at the National Synchrotron Light Source II (NSLS-II), Brookhaven National Laboratory. The energy of the beam was $13.5 \mathrm{keV}$, which corresponds to a wavelength of $0.9814 \AA$. Data were collected from $q$ $=3.13 \times 10^{-3}$ to $q=1.67 \times 10^{-1} \AA^{-1}$. For X-ray scattering measurements, a small excised piece of each film sample was placed on a silica wafer on an aluminum stage. Samples were measured at room temperature, and under vacuum to reduce background noise. Measurements through the film depth were obtained by incrementally translating the sample through a microfocused X-ray beam (full-width half-maximum of $26.6 \mu \mathrm{m}$ ), in $10 \mu \mathrm{m}$ steps, starting slightly below the filmsubstrate interface. Each X-ray scattering image was collected for 30 s or 120 s, yielding sufficient signal-to-noise for the particle form factor oscillations to be resolved.

\section{Results and Discussion}

Representative SAXS data on mixed films are presented in Figure 2 as offset stacked 1D curves, along with a representative 2D image for each sample. The positions in the direction normal to the film, $z$, have been normalized on the total height of the dry films, $H_{d r y}$, to obtain a scaled film depth, $\bar{z}=z / H_{d r y}$, which is 0.0 at the bottom of the film and 1.0 at the top of the film. Intensity data from pure silica and PS films are shown in Figure 3. For all samples, the intensity data are smooth over the measured $q$-range, with a slight increase in the noise for some samples for data points corresponding to the uppermost and lowest layers in the film. However, overall the experiments show good signal-to-noise with the protocol used for the microbeam experiments, giving us confidence in parameters derived from our analysis. As discussed further below, the broad peaks we observe can be ascribed to the lack of long-range order in these materials and are not due to the resolution of the beam (i.e., spread in the incident wavelength). 


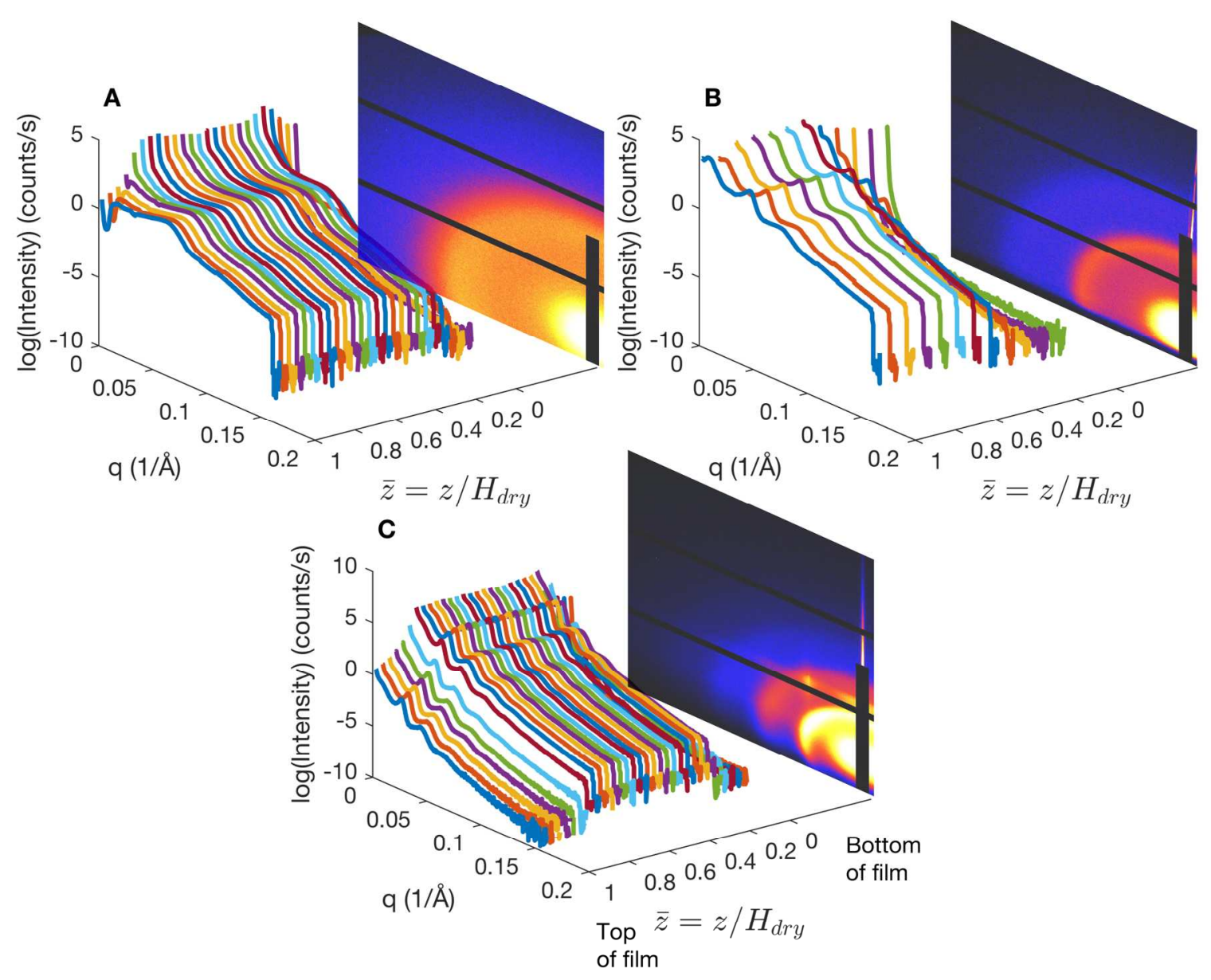

Figure 2: Offset stacked 1D curves for the scattered intensity of mixed films for different vertical depths. (A) PS-21 and S-8, $\alpha=2.6$; (B) PS-21 and S-10, $\alpha=2.2$; and (C) PS-21 and S-17, $\alpha=$ 1.2. Film positions have been scaled on the total height of the dry film. A representative $2 \mathrm{D}$ image for a single vertical position is also shown for each sample. Stacked 1D curves for these samples are shown in the Supporting Information (Figure S2). 

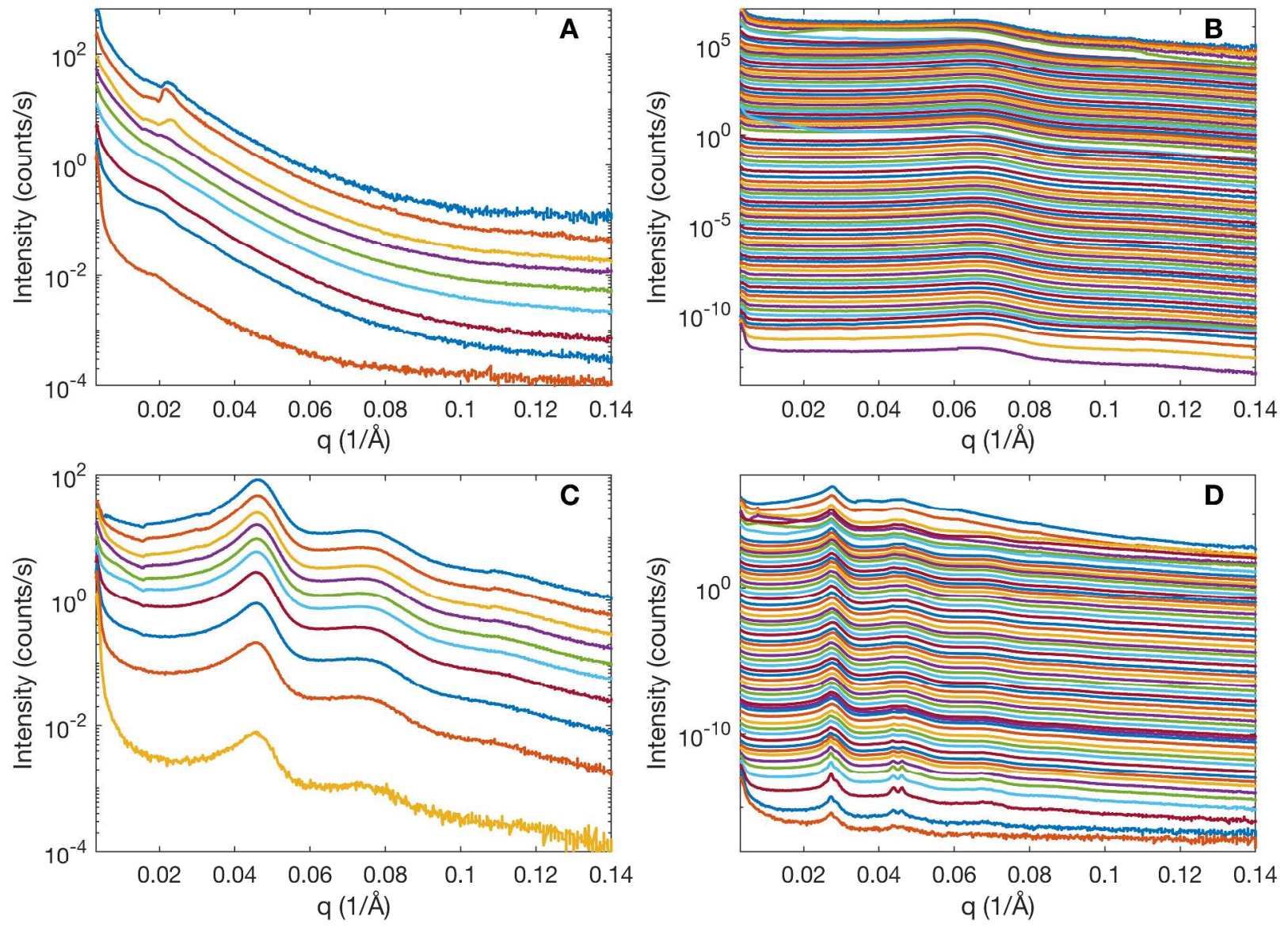

Figure 3. Scattered intensity data at different film depths for pure films, vertically shifted for clarity. After shifting, the upper curves in each graph corresponds to the top of the film, and the lowest curve to the bottom of the film. (A) Pure PS-21; (B) pure S-8; (C) pure S-10; and (D) pure S-17.

Pure films show similar scattering patterns at all film depths, indicating a relatively homogeneous structure (Figure 3). As expected for disordered packing of spherical objects, the scattering curves of the pure components exhibit weak and broad small-angle peaks, essentially the structure factor of a disordered material, encoding the preferred packing distance but without long-range order. Similarly, mixed films also shown broad peaks, indicative of disordered colloidal materials (Figure 2). An increase in intensity was observed at low $q$ at the bottom of the film, likely due to small-angle scattering from the film-substrate interface. These small deviations when the beam impinges on the film-substrate interface may be due to the scattering 
contribution of this interface, or the irregular edge of the substrate. Some of the mixed films showed changes in peak features as the depth changed, while others showed no significant changes.

We calculated the local PS and silica concentrations at different film depths using a linear combination analysis where the intensity of the mixed film at a fractional depth was assumed to be a weighted summation of the intensities of the pure PS and silica films at the same depth (Equation 1):

$I_{P S+S}(z)=a I_{P S}(z)+b I_{S}(z)$

We note that, strictly speaking, the scattering intensity of the mixed system should not be simple linear combination of the scattering curves of the constituent pure materials, because of the contribution of inter-type scattering. For instance, packing of two particle types can give rise to local configurations, and thus scattering signatures, not present in either pure system. However, our systems are disordered and do not show any signatures of long-range order. Additionally, this type of analysis may need to be modified for systems where solvent is present, as particles in those environments will likely have long-range interparticle interactions (e.g., electrostatic interactions) which may be affected by the presence of a second particle. Nevertheless, for disordered colloids in solvent-free conditions, such as those studied here, a linear combination of the pure material scattering curves provides a reasonable approximation to the total scattering curve. Scattering data was fit in the range $q=3.13 \times 10^{-3}$ to $q=1.67 \times 10^{-1} \AA$. Representative data displaying this fitting is provided in Figure 4. 


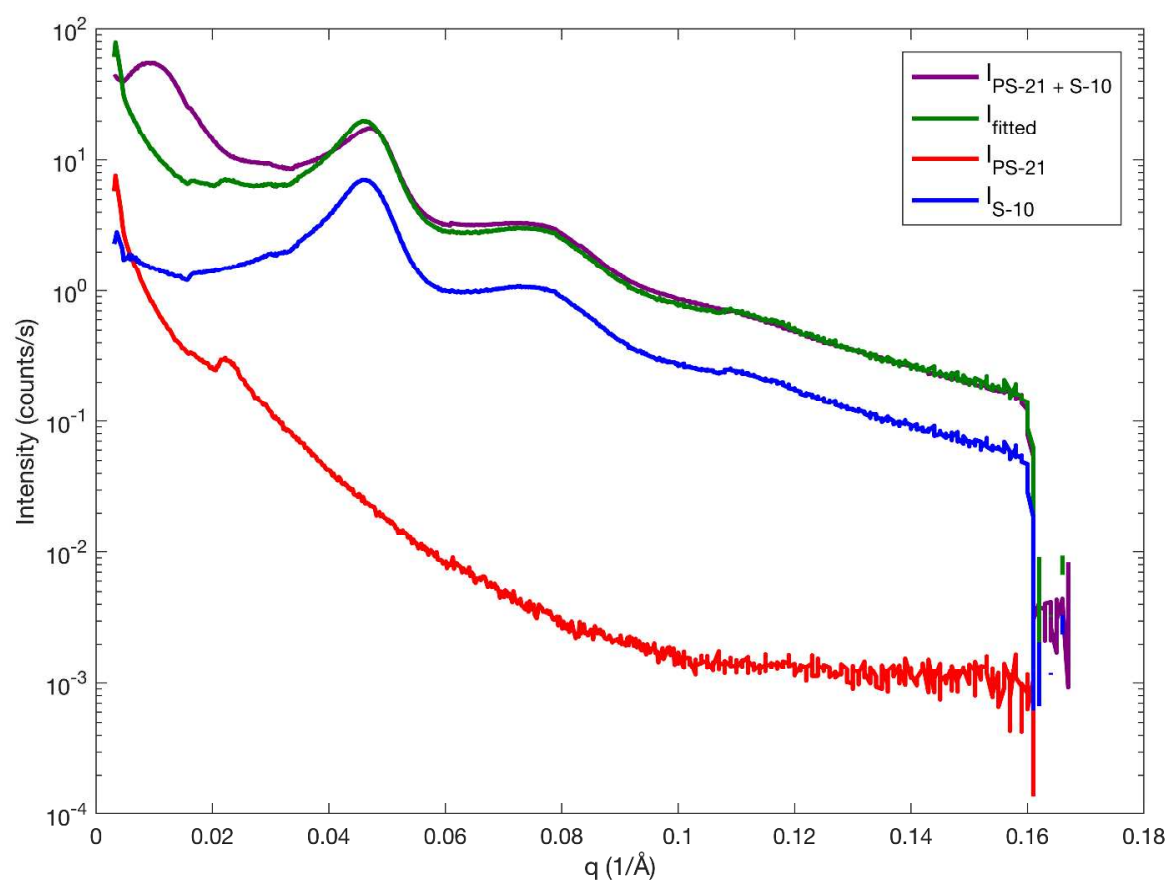

Figure 4: Representative scattering data for PS-21/S-10 sample (purple), mixed data fitting using Equation 1 (green), pure PS-21 (red), and pure S-10 (blue). All data are from the top of the film, i.e. $\bar{Z}=1.0$.

The factors for silica obtained from this analysis were then normalized to the bulk silica concentration to obtain a volume fraction. Local silica volume fractions as a function of film depth, which demonstrate stratification behavior, are shown in Figure 5. Layers with fits having a negative coefficient of determination, $R^{2}$, were eliminated from the analysis; at most, this resulted in 1-2 data sets from the top and bottom edges of the film being removed. Data near interfaces is expected to be less reliable owing to the scattering contribution of the film interfaces themselves. Relative errors in the silica volume fraction for the remaining data sets estimated from $R^{2}$ were less than $2 \%$, indicating good agreement between the data and model fit. However, the $R^{2}$ values are based on fits from large data sets (approximately 800 values of $I(q)$ are generated at each film position); thus, the uncertainty derived from a goodness-of-fit analysis may be underestimating the error. To provide a more conservative assessment of the uncertainty 
in our fit values of the volume fraction, we use the average uncertainty in $I$ for each film position, which we estimate as $I_{\text {err }}=\left[e r r_{\text {shot }}{ }^{2}+e r r_{\text {std }}{ }^{2}\right]^{1 / 2}$, where $e r r_{\text {shot }}$ is the Poisson-like "shot noise error" associated with photon counting statistics for a signal of a given intensity in the 1D average curve, and $e r r_{\text {std }}$ is the standard deviation of the 2D data along an arc at a particular $q$. This is likely an overestimate of the uncertainty in $I$, as it does not account for the fact $I$ has been averaged over multiple detector pixels and over the time of the measurement. Additionally, including both $e r r_{\text {shot }}$ and $e r r_{s t d}$ essentially double-counts some of the variation in $I$. Thus, this provides an upper-bound on the error in $I$. Using this approach, the relative error in $I$ was found to be approximately $4 \%$, and this is the relative error that was used for the volume fractions derived from our fits in Figure 5. Variations within concentration are reasonable due to local inhomogeneities as well as changes in surface roughness. Additionally, although cracking features did not develop until late in the drying process, they may still have impacted particle concentrations at some depths. Delamination may also have occurred and contribute to concentration variations. 

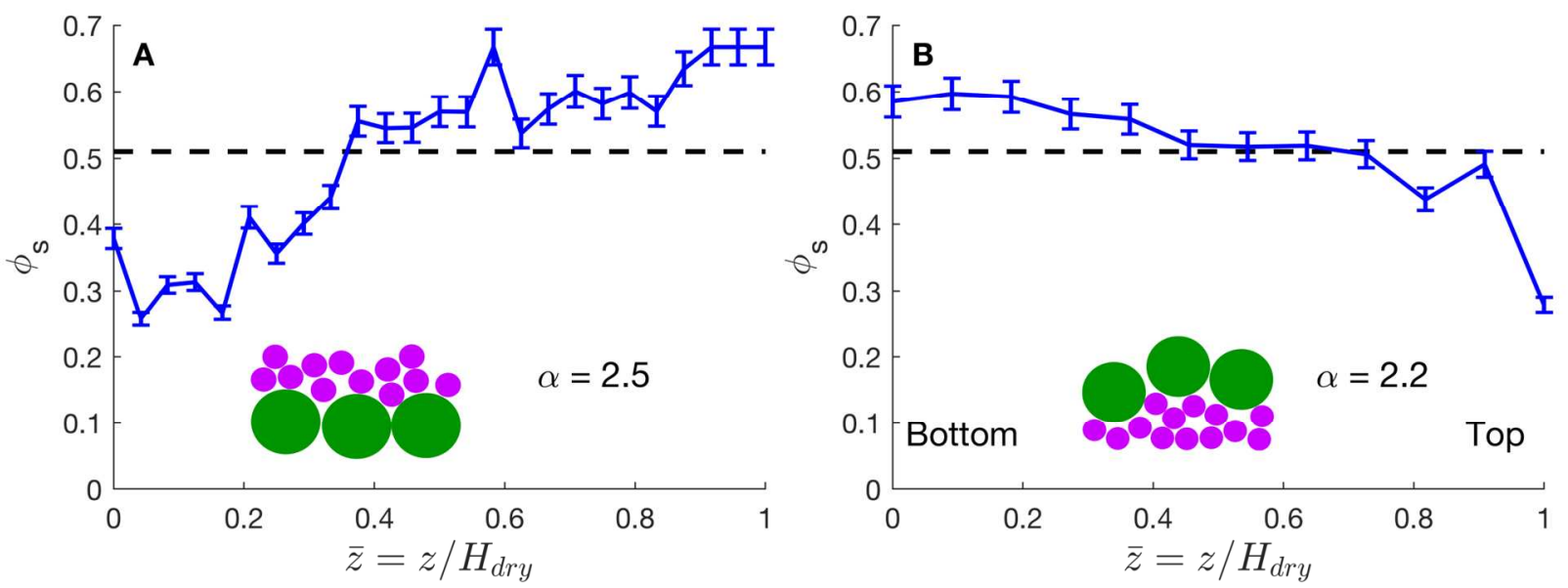

An inverted small-on-top configuration appears for the mixed PS-21 and small S-8 sample (Figure 5A, $\alpha=2.6$ ). The largest silica volume fraction obtained in this sample was 0.67 , which occurs near the top surface of this sample. As this is greater than the volume fraction for random close-packing, 0.64 , there may be some weak ordering of silica particles in these regions of the film. However, the SAXS spectra do not show sharp higher-order peaks, so any ordering that occurs is still fairly weak and short-range. Although our SAXS patterns do not show strong ordering, hexagonal close-packed structures for pure silica films been previously observed ${ }^{29-30}$, 
so it is not unreasonable to expect some weak ordering to occur in parts of the film that contain high concentrations of silica.

Large-on-top stratification appears in the mixed PS-21 and mid-sized S-10 sample $(\alpha=$ 2.2) (Figure 5B). While the change in volume fraction is not as large as the PS-21/S-8 sample, the general decrease in the concentration of silica as the film depth increases is still significant. The remaining mixed PS-21 and largest S-17 sample $(\alpha=1.2)$ (Figure 5C) displays no obvious stratification.

To aid in understanding the variation of stratification behavior observed in our systems, we compare our results to the state diagram proposed by $\mathrm{ZJD}^{24}$. Their work uses the parameter $\alpha^{2}\left(P e_{s}+1\right)$ to generate the following conditions: (a) large-on-top stratification will occur when $\alpha^{2}\left(1+P e_{s}\right)<\frac{C}{\phi_{s}}$; and (b) small-on-top stratification will occur when $\alpha^{2}\left(1+P e_{s}\right)>\frac{C}{\phi_{s}}$ where s denotes the small particle, $\phi$ is the volume fraction within the solvent, and $C$ is an arbitrary constant $^{24}$. They found good agreement between their stratification state diagram and theoretical results when $C=1$. It is important to note that more recent simulations have utilized models with an improved description of the particle hydrodynamics and interparticle interactions ${ }^{4,25-27}$ and with explicit solvent ${ }^{25,27}$. Specifically, results from $\mathrm{Sear}^{25}, \mathrm{SW}^{26}$, and Cheng and co-workers ${ }^{27}$ suggest that the model of ZJD may overpredict small-on-top stratification, and that the condition for small-on-top stratification is $P e_{s} \phi_{s} \gtrsim C$, where $C$ is found to be 0.64 by Sear ${ }^{25}, \sim 2$ by SW ${ }^{26}$, and $\sim 1$ by Cheng and co-workers ${ }^{27}$. Additionally, Sear derives the condition that no stratification occurs for $\phi_{s}>0.2$, although these results focus on the regime where the size ratio $\alpha$ is $>>1$, which is different than the regime used for our experiments.

Despite the limitations of the ZJD description highlighted by these more recent simulations, we still feel that a comparison with their state diagram is useful. Their state diagram 
has been derived for systems where the size ratio $\alpha$ is small, similar to our experimental conditions. Deviations we observe may provide insight into directions for future improvement in theoretical descriptions of stratification. Our data are compared to the stratification conditions of ZJD in Figure 6A. We find that $C=0.75$ provides better agreement with our experimental data than $C=1$. Our PS-21 and S-10 sample showing large-on-top stratification and our PS-21 and S8 sample displaying an inverted stratification both match the predictions of conditions (a) and (b). We plotted our remaining non-stratified sample for completeness, and as described further below, this result does not match the expectations of the ZJD state diagram.

The second set of state diagrams (Figure 6B-6D) considers stratification from a $P e_{\mathrm{s}}$ perspective. Here, ZJD present two conditions to describe stratification $\left.{ }^{24}: 1\right)$ no stratification will occur when $P e_{S}<\frac{1}{\alpha} ; 2$ ) large-on-top stratification will occur when $\frac{1}{\alpha}<P e_{S}<\frac{C}{\phi_{S} \alpha^{2}}-1$; and 3) small-on-top stratification will occur when $P e_{s}>\frac{c}{\phi_{s} \alpha^{2}}-1$. Some of our results agree with these analytically derived expressions and simulation results when $C=0.75$. Again, our large-on-top and small-on-top stratified samples match the predictions of conditions (2) and (3), respectively. According to the ZJD boundaries, our PS-21 and S-17 sample should yield large-on-top stratification, similar to that observed for PS-21 and S-10.

However, our results disagree with the condition of ZJD for no stratification. Interestingly, simulations of Cheng and co-workers ${ }^{27}$ show a regime of no stratification, mainly at higher $\phi_{s}$, although the $\phi_{s}$ at which the transition to no stratification occurs appears to depend upon the value of $P e_{s}$. This behavior is not predicted by either Sear or ZJD. As we did not vary $\phi_{s}$ in these experiments, we cannot test the predictions of Cheng and co-workers for no stratification. Work is ongoing to experimentally explore a larger parameter space for the specific case of no stratification. 
Some deviations between our results and those of ZJD are expected. As discussed above, recent simulations suggest that there are some limitations of this model and have provided improved descriptions of stratification that account hydrodynamics, interparticle interactions, and solvent effects. Additionally, we estimated the value of $C=0.75$ to be consistent with the data presented in Figure 6 as opposed to the value $C=1.0$ selected by ZJD. based on their results. Nevertheless, our results do provide some experimental confirmation of the existence of both small-on-top and large-on-top stratification behaviors with trends that qualitatively agree with the predictions of ZJD.
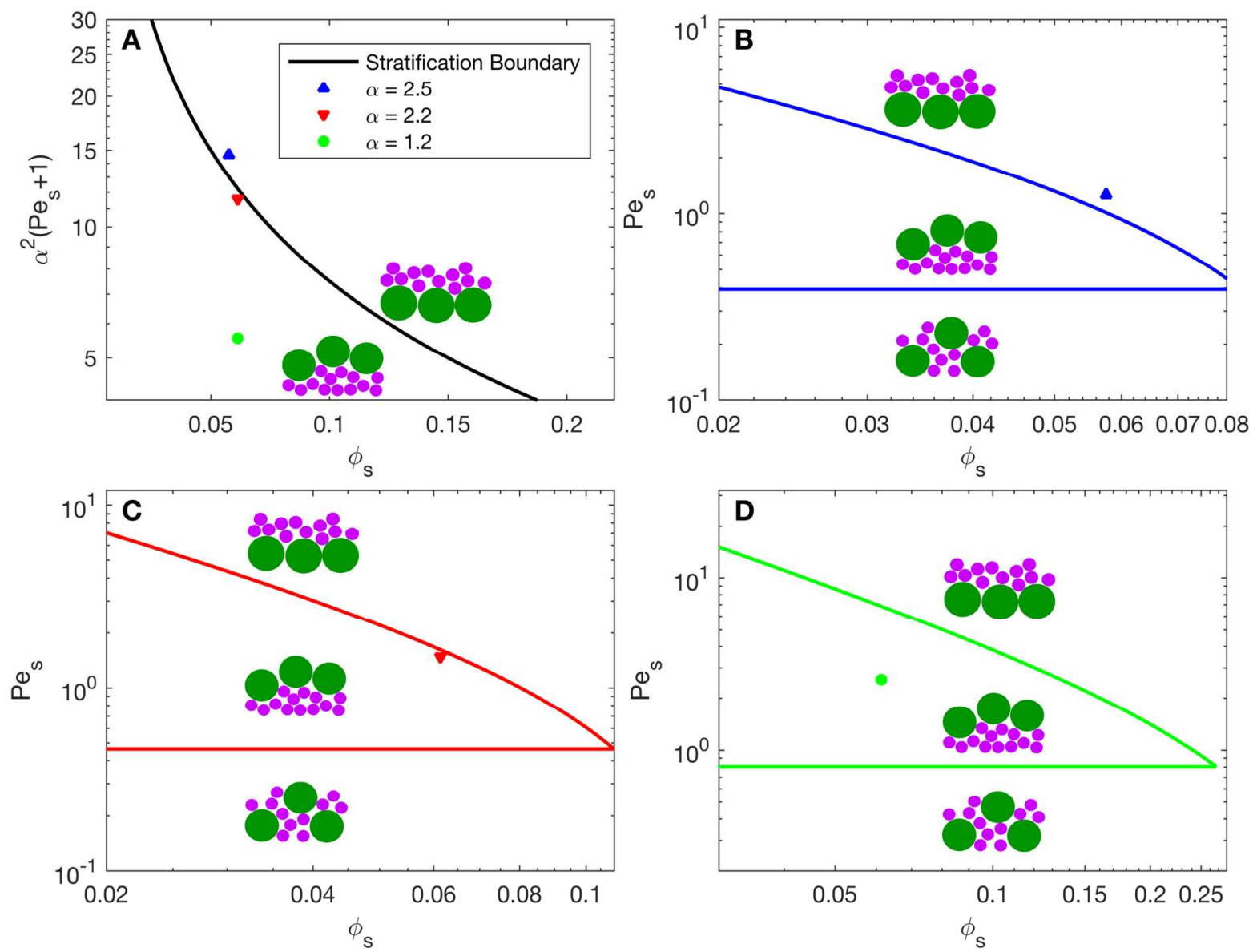

Figure 6: Plots of stratification state diagrams as presented by Zhou et al. ${ }^{24}$ compared to our experimental data. (A) Large-on-top versus small-on-top stratification boundary condition derived from $\alpha^{2}\left(P e_{s}+1\right)$ using $C=0.75$ where an upward-facing triangle shows small-on-top stratification, a downward-facing triangle shows large-on-top stratification, and a circle shows no stratification; (B), (C), and (D) each show non-stratified, large-on-top, and small-on-top 
boundary conditions generated from $P e_{\mathrm{S}}$ using $C=0.75$ for samples PS-21 and S-8 $(\alpha=2.6)$, PS21 and S-10 $(\alpha=2.2)$, and PS-21 and S-17 $(\alpha=1.2)$, respectively.

\section{Conclusions}

In summary, we present experimental verification of film stratification using microbeam SAXS. Our experimental technique is both novel and applicable to many types of binary particle systems and can provide quantitative local particle concentrations at different vertical depths. Samples utilizing three different sized small particles produced films within each of the three stratification regimes, i.e. no stratification, large-on-top, and small-on-top. The particle sizes and size ratios are smaller than those considered in previous works but still agree with some previously published results ${ }^{4,21-22,24}$. Our large-on-top results match the boundary conditions posited by Zhou et al. but have not, to our knowledge, been quantitatively experimentally observed using such a small $\alpha$. The no stratification results do not match predictions from existing state diagrams. Taken together, our data support some of the analytically derived stratification boundaries. Rigorous theory to explain and predict each regime is still needed.

\section{Supporting Information}

Plot of mass lost over time used within rate of evaporation calculation; vertically separated scattering intensity data over $q$ for film mixtures; form and structure factor fits for representative pure film samples.

\section{Acknowledgements}

Financial support for this work was provided by the National Science Foundation through award CBET-1335787 and a Department of Education Graduate Assistance in Areas of National Need (GAANN) fellowship for A. J. C., Award P200A160163. This research used beamline 11-BM, CMS, of the National Synchrotron Light Source II, a U.S. Department of Energy (DOE) Office 
of Science User Facility operated for the DOE Office of Science by Brookhaven National Laboratory under Contract No. DE-SC0012704.

\section{References}

1. Nikiforow, I.; Adams, J.; Konig, A. M.; Langhoff, A.; Pohl, K.; Turshatov, A.;

Johannsmann, D., Self-stratification during film formation from latex blends driven by differences in collective diffusivity. Langmuir 2010, 26, 13162-13167.

2. Atmuri, A. K.; Bhatia, S. R.; Routh, A. F., Autostratification in drying colloidal dispersions: effect of particle interactions. Langmuir 2012, 28, 2652-2658.

3. Zhang, C.; Zhao, X.; Lei, J.; Ma, Y.; Du, F., The wetting behavior of aqueous surfactant solutions on wheat (Triticum aestivum) leaf surfaces. Soft Matter 2017, 13, 503-513.

4. Howard, M. P.; Nikoubashman, A.; Panagiotopoulos, A. Z., Stratification dynamics in drying colloidal mixtures. Langmuir 2017, 33, 3685-3693.

5. van Dam, D. B.; Kuerten, J. G. M., Modeling the drying of ink-jet-printed structures and experimental verification. Langmuir 2008, 24, 582-589.

6. Huang, Y.; Li, W.; Qin, M.; Zhou, H.; Zhang, X.; Li, F.; Song, Y., Printable functional chips based on nanoparticle assembly. Small 2017, 13, 1503339.

7. Zhu, J.; Hersam, M. C., Assembly and electronic applications of colloidal nanomaterials. Advanced Materials 2017, 29, 1603895.

8. $\quad$ Rockstuhl, C.; Fahr, S.; Bittkau, K.; Beckers, T.; Carius, R.; Haug, F.-J.; Soderstrom, T.; Ballif, C.; Lederer, F., Comparison and optimization of randomly textured surfaces in thin-film solar cells. Opt. Express 2010, 18, A335-A342.

9. Wiersma, D. S., Disordered photonics. Nat. Photonics 2013, 7, 188-196. 
10. Gehan, T. S.; Bag, M.; Renna, L. A.; Shen, X.; Algaier, D. D.; Lahti, P. M.; Russell, T. P.; Venkataraman, D., Multiscale active layer morphologies for organic photovoltaics through self-assembly of nanospheres. Nano Lett. 2014, 14, 5238-5243.

11. Tekin, E.; Smith, P. J.; Schubert, U. S., Inkjet printing as a deposition and patterning tool for polymers and inorganic particles. Soft Matter 2008, 4, 703-713.

12. Faers, M. A.; Pontzen, R., Factors influencing the association between active ingredient and adjuvant in the leaf deposit of adjuvant-containing suspoemulsion formulations. Pest Manage. Sci. 2008, 64, 820-833.

13. Prevo, B. G.; Hwang, Y.; Velev, O. D., Convective assembly of antireflective silica coatings with controlled thickness and refractive index. Chem. Mater. 2005, 17, 3642-3651.

14. Krogman, K. C.; Druffel, T.; Sunkara, M. K., Anti-reflective optical coatings incorporating nanoparticles. Nanotechnology 2005, 16, S338.

15. Routh, A. F.; Zimmerman, W. B., Distribution of particles during solvent evaporation from films. Chem. Eng. Sci. 2004, 59, 2961-2968.

16. Gorce, J. P.; Bovey, D.; McDonald, P. J.; Palasz, P.; Taylor, D.; Keddie, J. L., Vertical water distribution during the drying of polymer films cast from aqueous emulsions. Eur. Phys. $J$. E: Soft Matter Biol. Phys. 2002, 8, 421-429.

17. Luo, H.; Cardinal, C. M.; Scriven, L. E.; Francis, L. F., Ceramic nanoparticle/monodisperse latex coatings. Langmuir 2008, 24, 5552-5561.

18. Ekanayake, P.; McDonald, P. J.; Keddie, J. L., An experimental test of the scaling prediction for the spatial distribution of water during the drying of colloidal films. Eur. Phys. J.: Spec. Top. 2009, 166, 21-27. 
19. Trueman, R. E.; Lago Domingues, E.; Emmett, S. N.; Murray, M. W.; Routh, A. F., Auto-stratification in drying colloidal dispersions: a diffusive model. J. Colloid Interface Sci. 2012, 377, 207-212.

20. Trueman, R. E.; Lago Domingues, E.; Emmett, S. N.; Murray, M. W.; Keddie, J. L.; Routh, A. F., Autostratification in drying colloidal dispersions: experimental investigations. Langmuir 2012, 28, 3420-3428.

21. Fortini, A.; Martin-Fabiani, I.; De La Haye, J. L.; Dugas, P. Y.; Lansalot, M.; D'Agosto, F.; Bourgeat-Lami, E.; Keddie, J. L.; Sear, R. P., Dynamic stratification in drying films of colloidal mixtures. Phys. Rev. Lett. 2016, 116, 118301.

22. Makepeace, D. K.; Fortini, A.; Markov, A.; Locatelli, P.; Lindsay, C.; Moorhouse, S.; Lind, R.; Sear, R. P.; Keddie, J. L., Stratification in binary colloidal polymer films: experiment and simulations. Soft Matter 2017, 13, 6969-6980.

23. Cheng, S.; Grest, G. S., Dispersing nanoparticles in a polymer film via solvent evaporation. ACS Macro Lett. 2016, 5, 694-698.

24. Zhou, J.; Jiang, Y.; Doi, M., Cross interaction drives stratification in drying film of binary colloidal mixtures. Phys. Rev. Lett. 2017, 118, 108002.

25. Sear, R. P., Stratification of mixtures in evaporating liquid films occurs only for a range of volume fractions of the smaller component. J. Chem. Phys. 2018, 148, 134909.

26. Sear, R. P.; Warren, P. B., Diffusiophoresis in nonadsorbing polymer solutions: The Asakura-Oosawa model and stratification in drying films. Phys. Rev. E 2017, 96, 062602.

27. Tang, Y.; Grest, G. S.; Cheng, S., Stratification in drying films containing bidisperse mixtures of nanoparticles. Langmuir 2018, 34, 7161-7170. 
28. Liu, X.; Liu, W.; Carr, A. J.; Santiago Vazquez, D.; Nykypanchuk, D.; Majewski, P. W.; Routh, A. F.; Bhatia, S. R., Stratification during evaporative assembly of multicomponent nanoparticle films. J. Colloid Interface Sci. 2018, 515, 70-77.

29. Juhue, D.; Wang, Y.; Lang, J.; Leung, O.-M.; Goh, M. C.; Winnik, M. A., Surfactant Exudation in the presence of a coalescing aid in latex films studied by atomic force microscopy. J. Polym. Sci., Part B: Polym. Phys. 1995, 33, 1123-1133.

30. Li, J.; Cabane, B.; Sztucki, M.; Gummel, J.; Goehring, L., Drying dip-coated colloidal films. Langmuir 2012, 28, 200-208. 


\section{Table of Contents Figure}

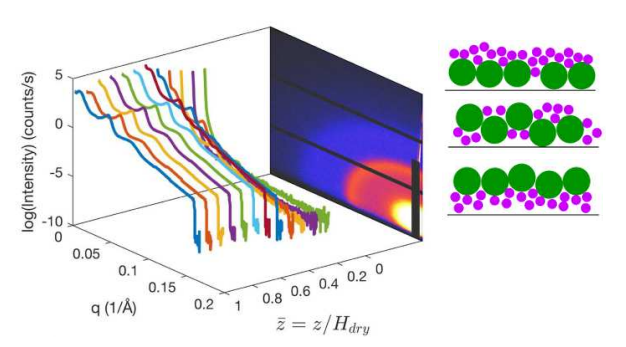

